\title{
Espectro polínico de mel de tiúba (Melipona fasciculata Smith, 1854, Hymenoptera, Apidae)
}

\author{
Adriana Cecília Lima MARTINS ${ }^{1}$, Márcia Maria Corrêa RÊGO², Léa Maria Medeiros CARREIRA³, \\ Patricia Maia Correia de ALBUQUERQUE ${ }^{4}$
}

\section{RESUMO}

O espectro polínico do mel de Melipona fasciculata Smith foi analisado com o objetivo de identificar os recursos nectaríferos utilizados por essa espécie. A identificação das plantas visitadas foi realizada com base na análise dos tipos polínicos encontrados em 12 amostras de mel coletadas, mensalmente, em uma colônia localizada no município de Palmeirândia, na área da Baixada Ocidental Maranhense (0240’47,6S, 4452’39,8”W), Brasil. As análises quantitativas e qualitativas foram realizadas com o objetivo de determinar as porcentagens e classes de frequência dos tipos polínicos presentes nas amostras de mel. Foram encontrados 45 tipos polínicos, sendo Pontederia parviflora Alexander (Pontederiaceae), espécie mais frequente em todo o período de amostragem (38,6\%), pólen dominante em Outubro (86\%), Junho (85\%), Julho (76\%), Agosto (49\%) e Setembro (51\%) e pólen acessório em Dezembro, Janeiro e Março. Mimosa caesalpiniifolia Benth (Mimosaceae) foi a segunda espécie mais frequente (22,8\%) sendo pólen dominante em Novembro (46\%), Abril (74\%) e Maio (72\%). Myrcia eximia DC (Myrtaceae) foi considerada pólen isolado importante. As famílias mais representativas no espectro polínico das amostras de mel foram Pontederiaceae e Mimosaceae. 50\% dos méis foram biflorais, havendo também méis monoflorais (41,7\%) e heteroflorais (8,3\%).

PALAVRAS-Chave: Abelhas-sem-ferrão, Baixada Maranhense, Brasil, Meliponina, Melissopalinologia.

\section{Pollen spectrum of honey of "tiúba" (Melipona fasciculata Smith, 1854, Hymenoptera, Apidae)}

\begin{abstract}
The pollen spectrum of honey of the Melipona fasciculata was analyzed aiming to elucidate the nectariferous resources used by this species. The identification of the visited plants was based upon the analysis of the pollen spectrum of 12 honey samples collected in one meliponarium located in Palmeirândia county, lowland area of Maranhão State, Brazil (0240’47,6S, 4452’39,8”W). The quantitative and qualitative analysis of honey samples was conducted in order to determine the pollen type percentages and frequency classes. Forty-five pollen types were found, being Pontederia parviflora Alexander (Pontederiaceae) the most frequent species during period of sampling (38.6\%) and considered dominant pollen in October (86\%), June (85\%), July (76\%), August (49\%) and September (51\%) and accessory pollen in December, January and March. Mimosa caesalpiniifolia Benth (Mimosaceae) was the second most frequent species (22.8\%) and was a dominant pollen in November (46\%), April (74\%) and May (72\%). Myrcia eximia DC. (Myrtaceae) was considered important isolated pollen. The most representative families found in the pollen spectrum of the honey samples were Pontederiaceae and Mimosaceae. 50\% of honey samples was bifloral, but there were also monofloral (41.7\%) and heterofloral (8.3\%) honeys.
\end{abstract}

KEYWORDS: Brazil, Maranhão lowlands, Meliponina, Melissopalynology, Stingless bee

\footnotetext{
1 Universidade Federal do Maranhão. acl_martins@hotmail.com

2 Universidade Federal do Maranhão.regommc@uol.com.br

3 Museu Paraense Emílio Goeldi. lea@museu-goeldi.br

${ }^{4}$ Universidade Federal do Maranhão. patemaia@hotmail.com
} 


\section{INTRODUÇÃO}

Os Meliponíneos são de grande importância econômica como agentes polinizadores, visando a manutenção de espécies vegetais, o equilíbrio ecológico nos ecossistemas e a produção de mel e geoprópolis (Kerr 1987). Portanto, a meliponicultura é uma atividade vital que gera renda ao homem do campo, além de permitir a manutenção das espécies de abelhas e plantas (Aidar 1996).

Dessa forma, conhecer as espécies vegetais utilizadas como fontes de pólen e néctar é de grande importância aos meliponicultores. As plantas visitadas para a coleta de néctar podem ser identificadas a partir da análise do pólen contido no mel (Barth 1970a, b, c; Louveaux et al. 1970), permitindo também a sua caracterização nectarífera e origem geográfica (Carvalho et al. 2001). Seguindo uma periodicidade mensal, as análises palinológicas fornecem um calendário floral apícola valioso (Luz et al. 2007).

No Maranhão, a criação da espécie Melipona fasciculata, conhecida popularmente como "tiúba", desempenha um importante papel econômico como fonte de renda, principalmente, para várias famílias de baixo poder aquisitivo do interior do Estado (Bezerra 2002). A maioria das colônias é transferida para caixas "caboclas" (caixas rústicas sem nenhuma padronização), outras, porém, são criadas no próprio tronco da árvore em que foram encontradas. $\mathrm{O}$ manejo dessas abelhas é feito com informaçóes que os criadores adquiriram e vão passando de geração a geração, sendo poucos os que recebem um apoio técnico especializado.

Atualmente, a "tiúba" corre um sério risco de extinção, principalmente pelo rápido desflorestamento que o Estado sofreu nos últimos anos (Bezerra 2002). Embora ela seja uma espécie amplamente manipulada pelo homem (Kerr et al. 2001), pouco se conhece sobre o seu hábito alimentar. Estudos $\operatorname{com} M$. fasciculata foram desenvolvidos por Kerr et al. (1986/87) em algumas localidades no Maranhão, e obtiveram como resultado uma listagem preliminar das espécies vegetais utilizadas como fontes de pólen e néctar. Apesar da maioria das análises melissopalinológicas de Meliponinae no Brasil ter sido realizada na região amazônica (Barth 2004), não se tem dados sobre o espectro polínico do mel de $M$. fasciculata. Nesse contexto, foi objetivo do presente trabalho identificar e quantificar os tipos polínicos presentes no mel de $M$. fasciculata e avaliar pela frequência polínica o potencial de cada espécie vegetal como fornecedora de néctar.

\section{MATERIAL E MÉTODOS}

O estudo foi realizado em um meliponário situado no município de Palmeirândia, Baixada Ocidental Maranhense (0240’47,6”S, 4452’39,8’W), Brasil.
A Baixada Ocidental Maranhense é caracterizada por apresentar dois períodos climáticos bem definidos: um seco e um chuvoso. No período de maior incidência de chuva há formação de campos inundados periodicamente e a presença de espécies vegetais típicas de áreas alagadas como: Neptunia plena Benth (tripa de vaca), Pontederia parviflora Alexander (cebola) e Eichornia azurea. (Sw.) Kunth (aguapé) (Ibañes et al. 2000). Nas partes altas, livres de alagação demorada, e nos tesos (ilhas não inundáveis com dimensóes variadas), a vegetação é típica de floresta mista em meio da qual se destaca o "babaçu" (Orbignya phalerata Mart.) (Oliveira-Pereira e Rebêlo 2000).

Nas proximidades do meliponário, a vegetação caracterizase pela presença de culturas tais como: Byrsonima crassifolia L. Kunth (murici); Psidium guajava L. (goiaba); Malpighia glabra L. (acerola); Tamarindus indica L. (tamarindo); Bixa orellana L. (urucum); Cucumis anguria L. (maxixe); Manihot esculenta Crantz (macaxeira); Manihot utilissima Pohl (mandioca); Zea mays L. (milho) e Phaseolus vulgaris L. (feijão), além de plantas ruderais como Turnera ulmifolia L.; Borreria verticillata Mayer; Crotalaria retusa L.; Dalechampia scandens L., entre outras. No entorno, encontramos floresta secundária com capoeira e babaçuais; floresta mista com espécies de sucessão secundária e espécies de cerrado; e matas de corte seletivo com espécies como "sapucaia" (Lecythis pisonis L.), "jatobá" (Hymenaea courbaril L.) e "matamatá" (Eschweilera ovata (Cambess.) Miers.).

A pecuária de subsistência, principalmente a bubalinocultura, além da pesca são as principais atividades econômicas da região (Feitosa e Trovão 2006).

No período de Outubro de 2006 a Setembro de 2007 foram coletadas amostras de mel de $M$. fasciculata em uma colônia instalada em caixa cabocla. Dessas amostras foram retirados $10 \mathrm{~mL}$ de $\mathrm{mel}$, de potes abertos, que depois de diluídos em $20 \mathrm{~mL}$ de água destilada foram centrifugados por 5 minutos a $2000 \mathrm{rpm}$ (Louveaux et al. 1970). O sobrenadante foi descartado e o sedimento depositado no fundo do tubo de ensaio submetido ao método de acetólise (Erdtman 1960). O material acetolizado foi então montado em lâminas permanentes com gelatina glicerinada e lutado com parafina.

A identificação dos tipos polínicos foi realizada por comparação com as lâminas de referência, confeccionadas a partir de botóes florais das espécies vegetais encontradas no entorno do meliponário e com a literatura especializada. De cada amostra foram contados 1000 grãos de pólen e os resultados expressos em porcentagem, de acordo com a seguinte classificação: pólen dominante (>45\%); pólen acessório (16-45\%); pólen isolado importante (3-15\%) e pólen isolado ocasional (<3\%) (Barth 1970a, b, c; Louveaux et al. 1970). As lâminas encontram-se depositadas no laminário 
de referência do Laboratório de Estudos sobre Abelhas (Lea), da Universidade Federal do Maranhão.

\section{RESULTADOS E DISCUSSÃO}

Foram identificados nas amostras de mel de Melipona fasciculata 45 tipos polínicos distribuídos em 36 gêneros e 22 famílias (Tabela 1). Caesalpiniaceae e Myrtaceae apresentaram maior representatividade quanto ao número de tipos polínicos (6); Pontederiaceae (43,83\%) e Mimosaceae (27,25\%) apresentaram os tipos polínicos com maior frequência (Figura 1).

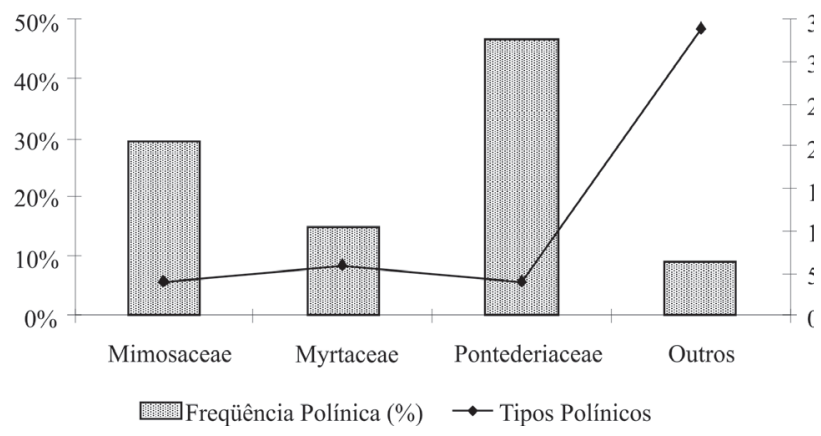

Figura 1 - Distribuição de tipos polínicos por família e frequência polínica baseada na Soma Polínica Total encontrada em amostras de méis de Melipona fasciculata no período de outubro de 2006 a setembro de 2007 no Município de Palmeirândia, Baixada Ocidental Maranhense.
Membros de Pontederiaceae têm uma ampla distribuição tropical, concentrando-se nos neotrópicos, particularmente no Brasil; são plantas muito abundantes em beiras de lagos, canais ou campos permanentemente alagados (Barret e Graham 1997). Flores dessa família são largamente polinizadas por abelhas de língua longa e têm o néctar como recurso primário (Husband e Barrett 1992).

Nas amostras de mel foram encontrados quatro representantes da família Pontederiaceae: Eichornia azurea, Eichornia crassipes, Pontederia sp. e Pontederia parviflora (Figura 2A), sendo a última a espécie mais frequente $(38,6 \%)$ em todo o período de amostragem. O pólen de $P$. parviflora foi dominante em cinco meses: Outubro (86\%); Junho (85\%); Julho (76\%); Agosto (49\%) e Setembro (51\%) e pólen acessório em Dezembro (40\%); Janeiro (29\%) e Março (30\%) (Tabela 1). Segundo Barth (2005), as plantas nectaríferas são de maior importância na produção de mel, apesar de que certa porcentagem do pólen encontrado no mel poder ser também de plantas anemófilas e poliníferas.

No período chuvoso ocorre o extravasamento dos grandes rios e os campos sofrem inundaçóes, permanecendo alagados em parte do ano (Araújo et al. 2003). Nessa regiáo encontramos plantas típicas de áreas alagadas como: Neptunia plena (tripa de vaca), Eichornia azurea (aguapé) e Pontederia parviflora conhecida localmente como "cebola". Os moradores

Tabela 1 - Frequência relativa baseada na Soma Polínica Total dos tipos polínicos encontrados em amostras de mel de Melipona fasciculata no período de outubro de 2006 a setembro de 2007, município de Palmeirândia, Baixada Ocidental Maranhense.

\begin{tabular}{|c|c|c|c|c|c|c|c|c|c|c|c|c|c|c|}
\hline \multirow{2}{*}{ Família } & \multirow{2}{*}{ Tipos Polínicos } & Out & Nov & Dez & Jan & Fev & Mar & Abr & Mai & Jun & Jul & Ago & Set & \%Total / \\
\hline & & $\%$ & $\%$ & $\%$ & $\%$ & $\%$ & $\%$ & $\%$ & $\%$ & $\%$ & $\%$ & $\%$ & $\%$ & Família \\
\hline \multirow[t]{3}{*}{ Anacardiaceae } & & & & & & & & & & & & & & 0,67 \\
\hline & Anarcadium microcarpum Ducke & & & & & & & & 3 & & & & & \\
\hline & Tapirira guianensis. Aubl. & & & & & 3 & 2 & & & & & & & \\
\hline \multirow[t]{2}{*}{ Arecaceae } & & & & & & & & & & & & & & 0,08 \\
\hline & Orbignya phalerata Mart. & & & 1 & & & & & & & & & & \\
\hline \multirow{3}{*}{ Asteraceae } & & & & & & & & & & & & & & 0,25 \\
\hline & Mikania sp. & & & 1 & & & 1 & & & & & & & \\
\hline & Wulffia baccata (L.) Kuntze. & & & & & & 1 & & & & & & & \\
\hline \multirow[t]{2}{*}{ Bixaceae } & & & & & & & & & & & & & & 0,83 \\
\hline & Bixa orellana L. & & & 2 & 1 & 1 & & 1 & 1 & 2 & & 1 & 1 & \\
\hline \multirow[t]{2}{*}{ Burseraceae } & & & & & & & & & & & & & & 0,25 \\
\hline & Protium sp. & & & & 2 & & 1 & & & & & & & \\
\hline \multirow[t]{7}{*}{ Caesalpiniaceae } & & & & & & & & & & & & & & 2,58 \\
\hline & Cassia sp. & & & & & & & & & & & & 1 & \\
\hline & Hymenaea sp. & & & & & & & & & & & & 1 & \\
\hline & Recordoxylon sp. & & & & 2 & & & & & & & & & \\
\hline & Senna alata (L.) Roxb. & 1 & & 2 & & & 1 & & 1 & 1 & 1 & 3 & 6 & \\
\hline & Senna sp. & & 5 & 1 & 2 & & & & & 1 & & & & \\
\hline & Tamarindus indicus L. & & & & & & & 2 & & & & & & \\
\hline \multirow[t]{2}{*}{ Caparidaceae } & & & & & & & & & & & & & & 0,33 \\
\hline & Cleome spinosa Jacq. & & & & & & & & & & & & 4 & \\
\hline
\end{tabular}


ACTA

AMAZONICA

Espectro polínico de mel de tiúba (Melipona fasciculata Smith, 1854, Hymenoptera, Apidae)

Tabela 1 - Continuação

\begin{tabular}{|c|c|c|c|c|c|c|c|c|c|c|c|c|c|c|}
\hline \multirow{2}{*}{ Família } & \multirow{2}{*}{ Tipos Polínicos } & Out & Nov & Dez & Jan & Fev & Mar & Abr & Mai & Jun & Jul & Ago & Set & \%Total / \\
\hline & & $\%$ & $\%$ & $\%$ & $\%$ & $\%$ & $\%$ & $\%$ & $\%$ & $\%$ & $\%$ & $\%$ & $\%$ & Família \\
\hline \multirow[t]{2}{*}{ Cochlospermaceae } & & & & & & & & & & & & & & 0,42 \\
\hline & Cochlospermum sp. & & & & & & & & 5 & & & & & \\
\hline \multirow[t]{2}{*}{ Euphorbiaceae } & & & & & & & & & & & & & & 0,25 \\
\hline & Croton pullei Lanj. & & & & & & & & 2 & 1 & & & & \\
\hline \multirow[t]{2}{*}{ Lacistemaceae } & & & & & & & & & & & & & & 2,83 \\
\hline & Lacistema sp. & & & & & & 16 & 17 & 1 & & & & & \\
\hline \multirow[t]{2}{*}{ Lamiaceae } & & & & & & & & & & & & & & 0,08 \\
\hline & Hyptis sp. & & & & & & & & & & & 1 & & \\
\hline \multirow[t]{2}{*}{ Lecytidaceae } & & & & & & & & & & & & & & 0,5 \\
\hline & Gustavia augusta L. & & & 6 & & & & & & & & & & \\
\hline \multirow[t]{2}{*}{ Malpighiaceae } & & & & & & & & & & & & & & 0,08 \\
\hline & Tetrapteris sp. & & & & & & & & & & & & 1 & \\
\hline \multirow[t]{4}{*}{ Melastomataceae } & & & & & & & & & & & & & & 2 \\
\hline & Mouriri sp. & & & & 1 & 1 & 1 & & & & & & 3 & \\
\hline & Pterolepsis sp. & & & & & & & & 2 & & 4 & 6 & 3 & \\
\hline & Tibouchina sp. & & & 1 & & & & & 2 & & & & & \\
\hline \multirow[t]{5}{*}{ Mimosaceae } & & & & & & & & & & & & & & 27,25 \\
\hline & Entada sp. & & & & 1 & & 2 & & & & & & 10 & \\
\hline & Mimosa caesalpiniifolia Benth. & & 46 & 19 & 1 & 1 & 14 & 74 & 72 & 7 & 4 & 27 & 8 & \\
\hline & Mimosa pudica L. & 1 & 36 & 1 & & & & & & & & 1 & & \\
\hline & Neptunia plena (L.) Benth. & 1 & & & & & & & & & & & 1 & \\
\hline \multirow[t]{7}{*}{ Myrtaceae } & & & & & & & & & & & & & & 12,08 \\
\hline & Eugenia sp. & & 1 & & 10 & 8 & 7 & & & & & & & \\
\hline & Eugenia sp.2 & & & & 1 & 17 & & & & & & & & \\
\hline & Myrcia eximia DC. & & 1 & 5 & 26 & 42 & & & & & & & & \\
\hline & Myrcia fallax (Rich.) DC. & & & & & & 4 & & & & & & & \\
\hline & Myrcia silvatica (Mey.) DC. & & & & & 15 & 4 & & & & & & & \\
\hline & Psidium guajava L. & & 2 & & 2 & & & & & & & & & \\
\hline \multirow[t]{2}{*}{ Ochnaceae } & & & & & & & & & & & & & & 0,5 \\
\hline & Ouratea castanaefolia (DC.) Engl. & & & 4 & 1 & & & & & & & & 1 & \\
\hline \multirow[t]{2}{*}{ Oxalidaceae } & & & & & & & & & & & & & & 1,42 \\
\hline & Oxalis juruensis Diels. & 1 & & 5 & 5 & 1 & 5 & & & & & & & \\
\hline \multirow[t]{5}{*}{ Pontederiaceae } & & & & & & & & & & & & & & 43,83 \\
\hline & Eichornia azurea (Sw.) & 10 & 1 & 9 & 1 & 1 & 4 & & & 2 & 10 & 9 & 4 & \\
\hline & Eichornia crassipes (Mart.) Solms. & & & 1 & & & 1 & & & & 3 & 1 & & \\
\hline & Pontederia sp. & & & 1 & & & 1 & & & 1 & 1 & 1 & 1 & \\
\hline & Pontederia parviflora Alexander. & 86 & 5 & 40 & 29 & 5 & 30 & & 7 & 85 & 76 & 49 & 51 & \\
\hline \multirow[t]{2}{*}{ Rubiaceae } & & & & & & & & & & & & & & 0,17 \\
\hline & Spermacoce tenella Kunth & & & 1 & 1 & & & & & & & & & \\
\hline \multirow[t]{3}{*}{ Solanaceae } & & & & & & & & & & & & & & 2,75 \\
\hline & Solanum grandiflorum Ruiz \& Pav. & & & & 5 & 3 & & 6 & & & & & 2 & \\
\hline & Solanum juripeba Rich. & & 1 & & 9 & 1 & 4 & & 1 & & & & 1 & \\
\hline \multirow[t]{3}{*}{ Verbenaceae } & & & & & & & & & & & & & & 0,25 \\
\hline & Lantana camara L. & & & & & & 1 & & & & 1 & & & \\
\hline & Vitex triflora Vahl & & & & & & & & & & & & 1 & \\
\hline Indeterminado & & & 2 & & & 1 & & & 3 & & & 1 & & 0,58 \\
\hline & & 100 & 100 & 100 & 100 & 100 & 100 & 100 & 100 & 100 & 100 & 100 & 100 & 100 \\
\hline
\end{tabular}



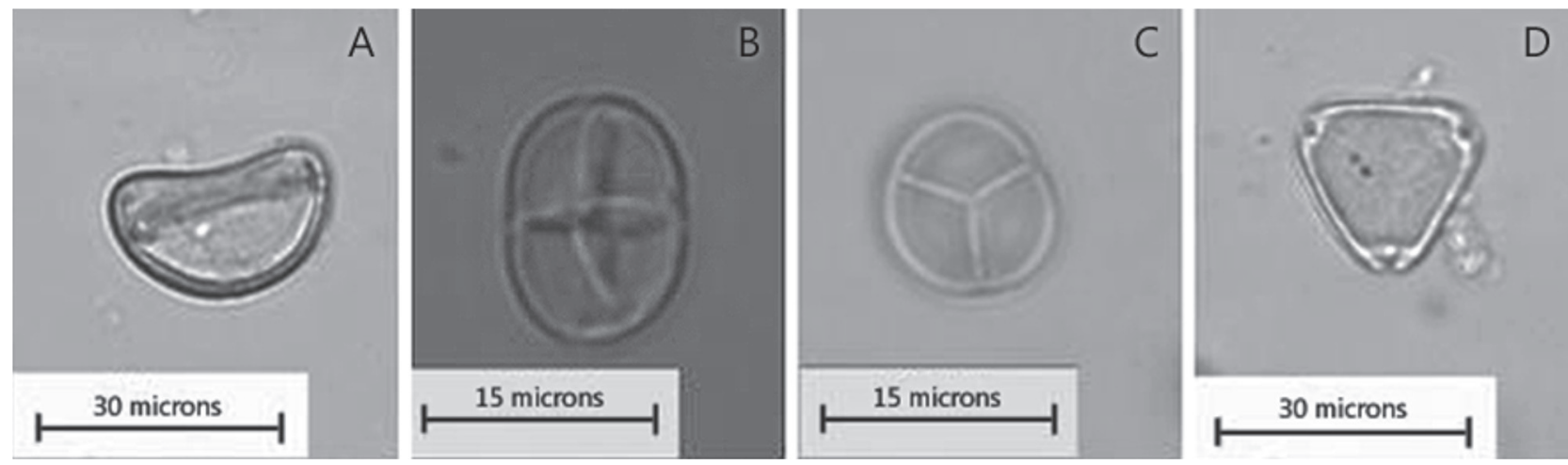

Figura 2 - Fotomicrografia dos principais tipos polínicos presentes em amostras de mel de Melipona fasciculata, provenientes do município de Palmerândia-MA: A - Pontederia parviflora (500x), B - Mimosa caesalpiniifolia (1000x); C - Mimosa pudica (1000x); D - Myrcia eximia (500x).

locais afirmam que a diminuiçáo da ocorrência de P. parviflora pela ação dos búfalos foi um dos fatores determinantes para o decréscimo da produção do mel, o que de certa forma pode ser verificado, já que em áreas pouco sujeitas à ação dos búfalos forma-se uma grande concentração desta espécie.

Ramalho et al. (1990), em sua revisão sobre espécies vegetais importantes, como fornecedoras de pólen e néctar para meliponíneos e abelhas africanizadas, ainda não haviam registrado nenhum representante de Pontederiaceae como sendo importante fornecedor desses recursos, o que pode ser justificado devido à maioria dos estudos realizados ter ocorrido em áreas sem influência de alagamentos. Já Pott e Pott (1986), apontaram Pontederia cordata e P. rotundifolia como importantes plantas aquáticas apícolas no Pantanal do Mato Grosso do Sul, tanto pela alta frequência com que ocorrem na regiáo quanto pela grande visitação das abelhas em suas flores, principalmente, na época de plena floração (de abril a agosto), no entanto, não avaliaram se são poliníferas ou/e nectaríferas. Em publicaçáo com base em informaçôes de herbário e levantamentos bibliográficos Salis et. al. (2009), incluíram várias espécies de Pontederia como importantes para Apis mellifera no Pantanal, inclusive P. parviflora florindo abundantemente de abril a maio e de agosto a setembro. Em nossos resultados as principais épocas de contribuição em néctar dessa espécie para o mel foram o inverno e a primavera (de junho a outubro), coincidindo com o período seco em Palmeirândia, o que demonstra que não depende das chuvas para florescer e que por isso se configura como uma constante fonte de recursos para Melipona fasciculata, disponível quase o ano todo.

Quanto a Mimosaceae, embora também tenha apresentado tipos polínicos de quatro espécies nas amostras de mel de $M$. fasciculata analisadas, apenas duas contribuíram de forma significativa na composição do mel (Tabela 1): Mimosa caesalpiniifolia (Figura 2B) e Mimosa pudica (Figura 2C). Muitas das espécies do gênero Mimosa são fontes de pólen ( $M$. scabrella), néctar ( $M$. bimucronata, $M$. invisa), ou ambos os recursos (M. dalleoides, M. pudica e M. velloziana) para os meliponíneos (Ramalho et al. 1990). Segundo Carrera et al. (1986) isso pode ser explicado devido a Mimosa ser uma planta ruderal, polinífera, ocorrendo em abundância em vários locais, além de grande parte florescer durante todo o ano, o que torna a família fonte inesgotável de recursos. Estudos realizados na Bahia revelaram a participaçáo de $M$. caesalpiniifolia na composição do mel de Melipona scutellaris Latreille e Melipona quadrifasciata Lepeletier (Carvalho et al. 2001, 2006). Barth (1970) ao analisar méis de Apis mellifera considera que muitas vezes o pólen de $M$. caesalpiniifolia é super-representado em relação à quantidade de néctar produzido uma vez que esta espécie é, principalmente, polinífera. Com relaçáo a $M$. pudica, embora sua frequência anual tenha sido baixa $(3,25 \%)$, teve uma importante contribuição no mês de Novembro, sendo responsável por $36 \%$ da frequência neste período.

No que diz respeito às demais famílias, a maioria das espécies identificadas apresentaram uma frequência muita baixa, sendo, portanto, classificadas como pólen isolado ocasional. Apenas Myrcia eximia (6,2\%) (Figura 2D), Eichornia azurea (4,3\%) e Mimosa pudica (3,3\%) foram classificadas como pólen isolado importante. Segundo Barth (1989), o pólen considerado ocasional tem pouca importância quanto à quantidade de néctar fornecido, embora possa ser de interesse na determinação da origem geográfica. Muitas das vezes os grãos de pólen com frequência muito baixa podem entrar na composição do mel de forma acidental, o que provavelmente ocorreu com Senna alata, já que Kiill et al. (2000), em trabalho com visitantes florais de plantas invasoras, concluíram que o único recurso fornecido por essa espécie vegetal é o pólen. Segundo Barth (1970), os méis brasileiros caracterizam-se pela grande quantidade de pólen na categoria isolado ocasional. 
A análise da composição mensal das amostras de mel demonstrou que cinco delas foram consideradas monoflorais em: Junho, Julho, Setembro e Outubro (mel de "cebola" - $P$. parviflora) e Maio (mel de "jamari”- M. caesalpiniifolia). Seis foram amostras biflorais em: Novembro (mel de "jamari”- $M$. caesalpiniifolia e "sensitiva" - M. pudica), Dezembro-Agosto (mel de "cebola" - P. parviflora e "jamari"- M. caesalpiniifolia), Janeiro (mel de "cebola" - P. parviflora e M. eximia), Março (mel de "cebola" - P. parviflora e Lacistema sp.) e Abril (mel de "jamari"- M. caesalpiniifolia e Lacistema sp.). A amostra do mel de Fevereiro foi heterofloral (mel de Eugenia sp. 2, Myrcia eximia e M. silvatica). Segundo Barth (1996), os méis monoflorais são característicos de áreas próximas a monoculturas, como o mel de Citrus e Eucalyptus. Para meliponíneos é comum que os méis sejam heteroflorais (Souza et al. 2006), mas há registros de méis monoflorais para Acacia polyphylla DC, Anadenanthera macrocarpa (Benth.), Citrus, Eucalyptus, Brassicaceae, M. caesalpiniifolia, Myrcia, Piptadenia rigida Benth, Schinus, Solanum e Vernonia polyanthes Less (Barth 2004).

Como pode ser observado, o mel de Melipona fasciculata apresentou na sua composição a presença do néctar de poucas espécies vegetais, sendo estas ruderais e nativas. Ramalho et al. (2007) afirmam que fatores como constância floral, restriçóes econômicas do forrageio, com ponto central fixo e troca de informaçóes sobre as fontes florais no interior das colônias, são fatores que podem explicar a concentração de atividade de forrageio dos Meliponina em poucas fontes florais. O mel de $M$. fasciculata em sua maioria foi classificado como monofloral, sendo Pontederia parviflora e Mimosa caesalpiniifolia as espécies mais frequentes. O conhecimento da flora apícola de uma região é uma importante ferramenta para a conservação das espécies vegetais e de abelhas, para elaboração de planos de manejo das espécies de abelhas, além de auxiliar o pequeno produtor na confecção do calendário apícola para um gerenciamento mais eficiente do meliponário.

\section{AGRADECIMENTOS}

Ao Sr. Miguel Roeder pelo apoio logístico recebido que permitiu realizar as primeiras viagens de coleta. À Professora Dra. Maria Lúcia Absy pelas sugestóes. A Carlos, Miguel, Dário, Cláudio Leandro e Fernanda pela companhia nas viagens de coleta. Ao Sr. Manoel Barros por permitir a execução do trabalho em seu meliponário. À Fundação de Amparo à Pesquisa do Estado do Maranhão (FAPEMA), e à Secretaria de Ciência e Tecnologia (SECTEC) pelo apoio logístico durante as viagens de coleta. À FAPEMA pela concessão da bolsa; aos consultores anônimos pelas valiosas sugestōes.

\section{BIBLIOGRAFIA CITADA}

Aidar, D.S.A. 1996. Mandaçaia: bee biology, management and artificial multiplication of colonies of Melipona quadrifasciata Lep (Hymenoptera, Apidae, Meliponinae. Brazilian Journal of Genetics. Monograps serie. 103 pp. (in Portuguese, with abstract in English).

Araújo, S.E.; Souza, S.R.; Fernandes, M.S. 2003. Morphological and molecular traits and accumulation of grain protein in rice varieties from Maranhão, Brazil. Pesquisa Agropecuária Brasileira, 38: 1281-1288. (in Portuguese, with abstract in English).

Barrett, S.C.H.; Graham, S.W. 1997. Adaptive radiation in the aquatic plant family Pontederiaceae: insights from phylogenetic analysis, p. 225-258. In: Givnish, T.J.; Sytsma, K. (Eds.) Molecular Evolution and Adaptive Radiation. Cambridge University Press. 638 pp.

Barth, O.M. 1970. Microscopic analysis of some samples of honey. 1. Dominant pollen. Anais da Academia Brasileira de Ciências, 42: 351-366. (in Portuguese).

Barth, O.M. 1970. Microscopic analysis of some samples of honey. 2. Acessories pollen. Anais da Academia Brasileira de Ciências, 42: 571-590. (in Portuguese).

Barth, O.M. 1970. Microscopic analysis of some samples of honey. 3. Isolated pollen. Anais da Academia Brasileira de Ciências, 42: 747-772. (in Portuguese).

Barth, O.M. 1989. The pollen in the Brazilian honey. Editora Luxor, Rio de Janeiro. 150 pp. (in Portuguese).

Barth, O.M. 1996. Monofloral and wild flower honey pollen espectra in Brazil. Ciencia e Cultura, 48: 163-166.

Barth, O.M. 2004. Melissopalynology in Brazil: a review of pollen analysis of honeys, própolis and pollen loads of bees. Scientia Agrícola, 61: 342-350.

Barth, O.M. 2005. Pollen analysis of honey: an evaluation of data and its meaning. Mensagem Doce, 81 (http://www.apacame.org. $\mathrm{br} / \mathrm{mensagemdoce/81/artigo.htm).} \mathrm{Acesso} \mathrm{em} \mathrm{08/07/2010.} \mathrm{(in}$ Portuguese).

Bezerra, M.D.B. 2002. Beekeeping, an essential activity to the household economy of the humid tropics, p. 144-203. In: Moura, E.G. de (Org.) Agro environments of transition: from the humid tropics and semi-arid. UEMA. 300 pp (in Portuguese).

Carreira, L.M.M.; Jardim, M.A.G.; Moura, C.O.; Pontes, M.A.; Marques, R.V. 1986. Pollen analysis of honeys from some municipalities of Pará. In: Proceedings of the 1st. International Symposium on the Humid Tropics, Belém (PA) 2: 79-84. (in Portuguese, with abstract in English).

Carvalho, C.A.L.; Moreti A.C.C.C.; Marchini, L.C.; Alves, R.M.O.; Oliveira, P.C.F.. 2001. Pollen spectrum of honey of "uruçu" bee (Melipona scutellaris Latreille, 1811). Revista Brasileira de Biologia, 61: 63-67.

Carvalho, C.A.L.; Nascimento, A.S.; Pereira, L.L.; Machado, C.M.; Clarton, L. 2006. Nectar and Pollen plants used by Melipona quadrifasciata (Hymenoptera: Apidae) in Recôncavo Baiano region, Brazil. Magistra. 18: 249-256. (in Portuguese, with abstract in English). 
Erdtman, G. 1960. The acetolysis method-a revised description. Svensk. Bot. Tidskr. 54: 541-550.

Feitosa, A.C.; Trovão, J.R. 2006. School Atlas of Maranhão: Geo-space and Cultural History. Ed. Grafset. 207 pp. (in Portuguese).

Husband, B.C.; Barrett, S.C.H. 1992. Effective population size and genetic drift in tristylous Eichhornia paniculata (Pontederiaceae). Evolution. 46: 1875-1890.

Ibañes, M.S.R; Calvacante, P.R.S.; Costa Neto, J.P.; Barbieri, R.; Pontes, J.P.; Santana, S.C.C.; Serra, C.L.M.; Nakamoto, N.; Mitamura, O. 2000. Limnological characteristics of three aquatic systems of the preamazonian floodplain, Baixada Maranhense (Maranhão, Brazil). Aquatic Ecosystem Health and Management, 3: 521-531.

Kerr, W.E. 1987. Brazilian native bees (stingless bees) for pollination, the production of honey, pollen, wax and geopropolis. Informe Agropecuário, 13: 15-22. (in Portuguese).

Kerr, W.E.; Absy, M.L.; Marques-Sousa, A.C. 1986/87. Nectar and polen species used by Melipona compressipes fasciculata bee, in Maranhão. Acta Amazonica, 16/17: 145-156. (in Portuguese, with abstract in English).

Kerr, W.E.; Carvalho, G.A.; Coletto-Silva, A.; Assis, M.G.P. 2001. Little Mentioned Aspects of Amazonian Biodiversity In: Ministério da Ciência e Tecnologia. Biodiversity, Research and Development in the Amazon, Strategic Partnerships, 12: 20-41. (in Portuguese, with abstract in English).

Kiill, L.H.P.; Haji, F.N.P.; Lima, P.C.F. 2000. Floral visitors of weed plants of irrigated orchard areas. Scientia Agricola. 57: 575-580. (in Portuguese, with abstract in English).

Louveaux, J.; Maurizio, A.; Vorwohl, G. 1970. Methodik der melissopalynologie. Apidologie, 1: 193-209.

Luz, C.F.P.; Thomé, M.L.; Barth, O.M. 2007. Trophic resources for Apis mellifera L. (Hymenoptera, Apidae) in the Morro Azul do Tinguá region, Rio de Janeiro state. Revista Brasileira de Botânica. 30: 29-36. (in Portuguese, with abstract in English).
Oliveira-Pereira, Y.N.; Rebelo, J.M.M. 2000. Species of Anopheles in Pinheiro municipality (Maranhão), endemic area of malaria. Revista da Sociedade Brasileira de Medicina Tropical, 33: 443-450. (in Portuguese, with abstract in English).

Pott, A.; Pott, V.J. 1986. Honey flora survey of the Pantanal, Mato grosso do Sul, Brazil. EMBRAPA-CPAP. Pesquisa em andamento, 3. 16 pp. (http://www.cpap.embrapa.br/publicacoes/online/ PA03.pdf) (in Portuguese, with abstract in English).

Ramalho, M.; Kleinert-Giovannini, A.; Imperatriz-Fonseca, V.L. 1990. Important bee plants for stingless bees (Melipona and Trigonini) and Africanized honeybees (Apis mellifera) in neotropical habitats: a review. Apidologie, 21: 469-488.

Ramalho, M.; Silva, M.D.; Carvalho, C.A.L. 2007. Harvesting Dynamics of Pollen Sources by Melipona scutellaris Latreille (Hymenoptera: Apidae): a Comparative Analysis with Apis mellifera L. (Hymenoptera: Apidae) in the Atlantic Forest Domain. Neotropical Entomology, 36: 38-45. (in Portuguese, with abstract in English).

Salis, M.S.; Reis, V.D.A.; Marcondes, A.N. 2009. Flowering of Bee Plants in the Pantanal Wetland Based on Herbarium and Literature Information. Corumbá: EMBRAPA Pantanal. Research and development bulletin. 46 pp. (http://www.cpap. embrapa.br/publicacoes/online/BP91.pdf). (in Portuguese, with abstract in English).

Souza, B.; Roubik, D.; Barth, M.O.; Heard, T.; Rnríquez, E.; Carvalho, C.; Villas-Bôas, J.; Marchini, L.; Locatelli, J.; PersanoOddo, L.; Almeida-Muradian, L.; Bogdanov, S.; Vit, P. 2006. Composition of Stingless bee Honey: Setting Quality Standards. Interciencia, 31: 867-875.

Recebido em 20/03/2010

Aceito em 31/10/2010 
